\title{
Ensayos en Ambiente Hospitalario de Técnicas de Monitorización de Bloqueo Neuromuscular: Resultados Preliminares
}

\author{
Trials of Neuromuscular Monitoring Blocking Techniques in Hospitals: \\ Preliminary Results \\ Recibido: 14 Mayo 2014/Enviado para modificación: 2 de Julio 2014/Aceptado: 17 de Julio 2014
}

\author{
Yadel Forneiro Martín Viaña ${ }^{1}$ \\ ICID - Tecnología Médica Digital \\ Daniel Vega Fernández ${ }^{2}$ \\ ICID - Tecnología Médica Digital \\ Idoris Cordero Escobar ${ }^{3}$ \\ Hospital Clínico Quirúrgico Hermanos Ameijeiras \\ Alberto Belisario Martínez Sardiñas ${ }^{4}$ \\ ICID - Tecnología Médica Digital \\ Yahima Sánchez Hernández ${ }^{5}$ \\ Hospital Clínico Quirúrgico Hermanos Ameijeiras \\ Liu Margarita Alfonso Zayas ${ }^{6}$ \\ ICID - Tecnologia Médica Digital
}

\section{RESUMEN}

Introducción: La respuesta de grupos musculares a consecuencia de la estimulación de nervios periféricos, permite a los anestesiólogos evaluar la profundidad del bloqueo neuromuscular durante la anestesia general. Objetivo: Evaluar en la práctica médica el prototipo de un monitor cubano de bloqueo neuromuscular y sus algoritmos, para calcular indicadores objetivos de relajación muscular. Materiales y métodos: Se escogieron cirugías de corta duración y se monitorizó la función neuromuscular intraoperatoria de pacientes. Los ensayos se realizaron a partir de la estimulación de los nervios mediano y cubital con el patrón Train-of-Four (TOF) a intensidad de corriente supramáxima, registrándose la respuesta cinética del dedo pulgar y calculando automáticamente los indicadores de relajación muscular. El protocolo fue aprobado por el Consejo Científico y el Comité de Ética del Hospital Clínico Quirúrgico "Hermanos Ameijeiras", en el marco de un proyecto de investigación conjunta entre el ICID -Tecnología Médica Digital y el Hospital. Resultados: Se presenta evidencia de la monitorización de cinco casos reales, para los que se construyeron gráficos del comportamiento en el tiempo de los indicadores de relajación muscular. El monitor sometido a ensayos se mantuvo funcionando de forma estable durante todos los actos quirúrgicos. Conclusión: A criterio de los especialistas involucrados, los indicadores de relajación muscular registrados durante los ensayos mostraron significativa correspondencia con la clínica de los pacientes, lo que proporciona evidencias a favor de las técnicas de monitorización implementadas.

Palabras Clave:Bloqueo neuromuscular, relajación muscular, monitorización intraoperatoria(Fuente: DeCS).

\section{ABSTRACT}

Introduction: In order to evaluate the magnitude of neuromuscular blocking during anesthesia, the anesthesiologists consider the muscular response to peripheral nerves stimulation. Objective: Study aims to calculate muscle relaxation indicators, through neuromuscular blocking and its algorithms using a Cuban made monitoring device during the medical procedure. Materials and methods: Thumb kinetic response and muscles relaxation indicators were registered and calculated automatically; for this purpose, median and nerves trials using Troin-of - Four (TOF) were conducted in shorter surgeries, which allowed to monitor patients' neuromuscular, intraoperative function. Scientific consel and the Committee of Ethics of the Clinical Surgical Hospital "HermanosAmeijeiras" approved the protocol, based on a research project conducted by the ICID - Medical Digital technology and the Hospital. Results: the monitoring was stable during all medical procedures as indicated on graph data obtained during five sample cases indicating muscle relaxation indicators. Conclusion: muscle relaxation indicators registered during trials showed significant relevance relation to patient's clinical history, giving support to evidence of the monitoring implemented techniques based on specialists' criteria.

Keywords:neuromuscular blocking, muscle relaxation, intraoperative monitoring (Source: MeSH, NLM).

Para citar este artículo: Martín VY, Vega FD, Cordero EI, Martínez SB, Sánchez HY, Alfonso ZL. Ensayos en ambiente hospitalario de técnicas de monitorización de bloqueo neuromuscular: resultados preliminares. Cienc. innov. salud. 2014; 2 (2):54 - 62

\footnotetext{
${ }^{1}$ Máster en Sistemas Digitales. ICID - Tecnología Médica Digital. Correo electrónico: yforneiro@ @icid.cu

${ }^{2}$ Máster en Sistemas Digitales. ICID - Tecnología Médica Digital.

${ }^{3}$ Médico. PhD. Especialista en Anestesiología y Reanimación. Hospital Clínico Quirúrgico Hermanos Ameijeiras.

${ }^{4}$ Médico. Especialista en Anestesiología y Reanimación. ICID - Tecnología Médica Digital.

${ }^{5}$ Médico. Residente de Anestesiología y Reanimación. Hospital Clínico Quirúrgico Hermanos Ameijeiras.

${ }^{6}$ Especialista General de la Electrónica. ICID - Tecnología Médica Digital.
} 


\section{Introducción}

Los Bloqueantes Neuromusculares (BNM) son fármacos de uso indispensable para la realización de variados procedimientos quirúrgicos, en los que es imprescindible la eliminación del tono muscular de los pacientes para facilitar el acceso quirúrgico.

La monitorización de la función neuromuscular en un paciente bajo la acción de un BNM es de gran importancia en la práctica anestesiológica, pues resulta necesario conocer el grado de relajación y el momento de ella en que se encuentran los pacientes. Esto permite al anestesiólogo tomar las decisiones adecuadas, tales como intubar o extubar al paciente para el inicio o fin de la respiración artificial; administrar dosis adicionales de BNM teniendo en cuenta las características y el curso de la intervención; y determinar el momento adecuado para revertir el proceso desencadenado por el BNM.

Uno de los métodos utilizados para la monitorización de la función neuromuscular consiste en la estimulación eléctrica de un nervio motor periférico y la valoración de la respuesta del músculo inervado por él (1-3). Entre las formas más novedosas de cuantificar esta respuesta, se halla la aceleromiografía, que consiste en adquirir información cinética (aceleración) del músculo inervado para cuantificar objetivamente el bloqueo neuromuscular (1-3).

La caracterización de la respuesta del dedo pulgar al aplicar el patrón TOF a los nervios mediano y cubital $(4,5)$, así como la obtención de criterios para la estimulación con intensidad supramáxima $(6,7)$ constituyen antecedentes importantes para el desarrollo y la implementación de un método y un monitor dirigidos a la monitorización objetiva de la función neuromuscular. Para su comprobación práctica, es imprescindible la realización de ensayos en casos quirúrgicos reales, porque a nivel de laboratorio no es permisible emplear BNM y no hay equipos simuladores de pacientes para este tipo de aplicación.
Por todo lo planteado, los objetivos de esta investigación son: evaluar en ambiente hospitalario y en casos quirúrgicos reales, el desempeño del prototipo funcional de un monitor autónomo de monitorización de bloqueo neuromuscular, la aceleromiografía y el algoritmo implementado en el monitor, para calcular indicadores objetivos de relajación muscular.

\section{Materiales y Métodos}

\section{A. Prototipo funcional del monitor de bloqueo neuromuscular}

Para los ensayos experimentales, se diseñó y construyó el prototipo funcional de un monitor de bloqueo neuromuscular que permitiera la aplicación del estímulo eléctrico al nervio aductor del pulgar, la captura sincronizada del movimiento mecánico del músculo inervado en términos de aceleración, y la ejecución del algoritmo para la obtención de los indicadores de relajación muscular $(8,9)$. Su diagrama en bloques aparece representado en la Figura 1.

El monitor puede ser energizado a través de una fuente de alimentación externa de grado médico o mediante baterías internas recargables. El teclado permite al operador el encendido del monitor, el ajuste de la intensidad de la corriente de estimulación eléctrica, la selección del patrón de estimulación y el ajuste del intervalo de tiempo entre eventos de monitorización, entre otras funciones. El visualizador gráfico muestra el resultado de los eventos de monitorización del paciente en forma cualitativa (patrón de barras) y cuantitativa (indicadores numéricos porcentuales), así como el patrón de estimulación seleccionado y el estado del temporizador de monitorización automática. El algoritmo para la obtención de los indicadores de relajación muscular se ejecuta en el microcontrolador, que configura de manera apropiada la fuente de corriente durante la estimulación y adquiere desde el acelerómetro el resultado de las mediciones durante la respuesta. Por último, el termómetro permite medir la temperatura periférica del paciente para prevenir la hipotermia. 


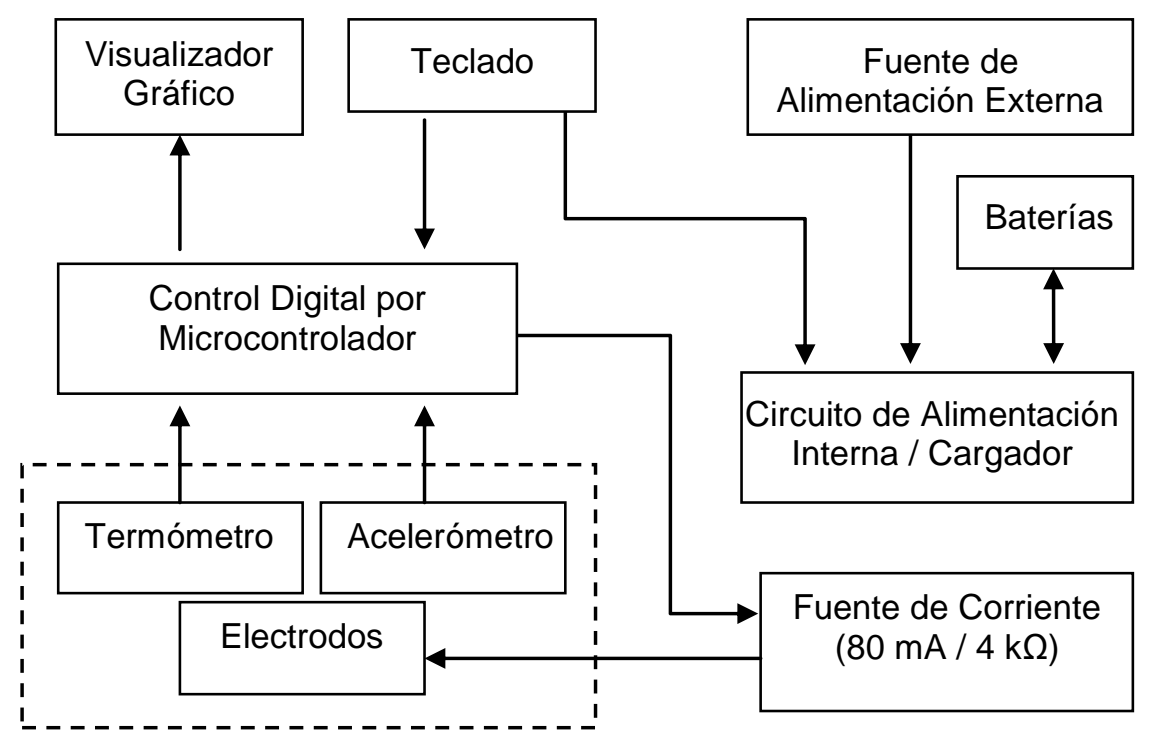

Figura 1. Diagrama en bloques del prototipo funcional del monitor de bloqueo neuromuscular. El acelerómetro, el termómetro y los electrodos constituyen partes aplicables al paciente.

\section{B. Patrones de estimulación eléctrica}

Para la realización de los ensayos, se incorporaron en el monitor dos patrones de estimulación conocidos en la práctica médica como Single Twitch (ST) y Train-of-Four(TOF) $(3,10)$. Las formas de onda de estos se representan en la Figura 2, en la cual se aprecia que: 1. Los parámetros de tiempo se encuentran predeterminados, siendo sólo ajustable la intensidad de los pulsos; 2. El patrón ST está formado por un estímulo simple monopolar y se emplea durante el proceso de calibración antes de la inserción del BNM, lo que permite obtener una referencia de la respuesta del paciente antes de la iniciación del acto quirúrgico; 3. El patrón TOF está constituido por una serie de cuatro pulsos monopolares, y se emplea durante los eventos de monitorización del paciente que tienen lugar en el acto quirúrgico.
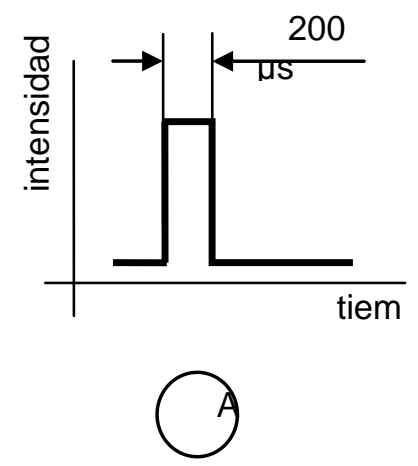
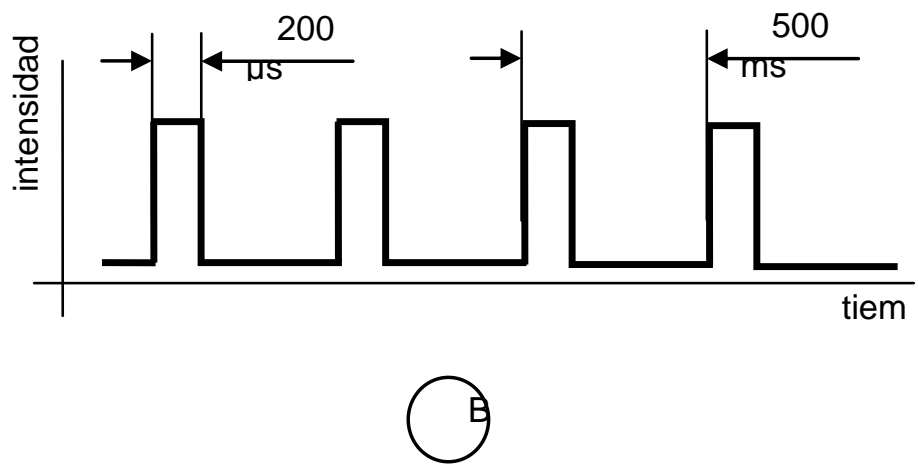

Figura 2: Patrones de estimulación eléctrica empleados en los ensayos. Se denota por " $A$ " el patrón Single Twitch (ST) y por "B" el patrón Train-of-Four(TOF).

\section{Algoritmo para la estimación del bloqueo neuromuscular}

El algoritmo implementado en el monitor para la estimación del bloqueo neuromuscular, que incluye el 
proceso de calibración y la ejecución de eventos de

monitorización, se representa en bloques en la Figura3.

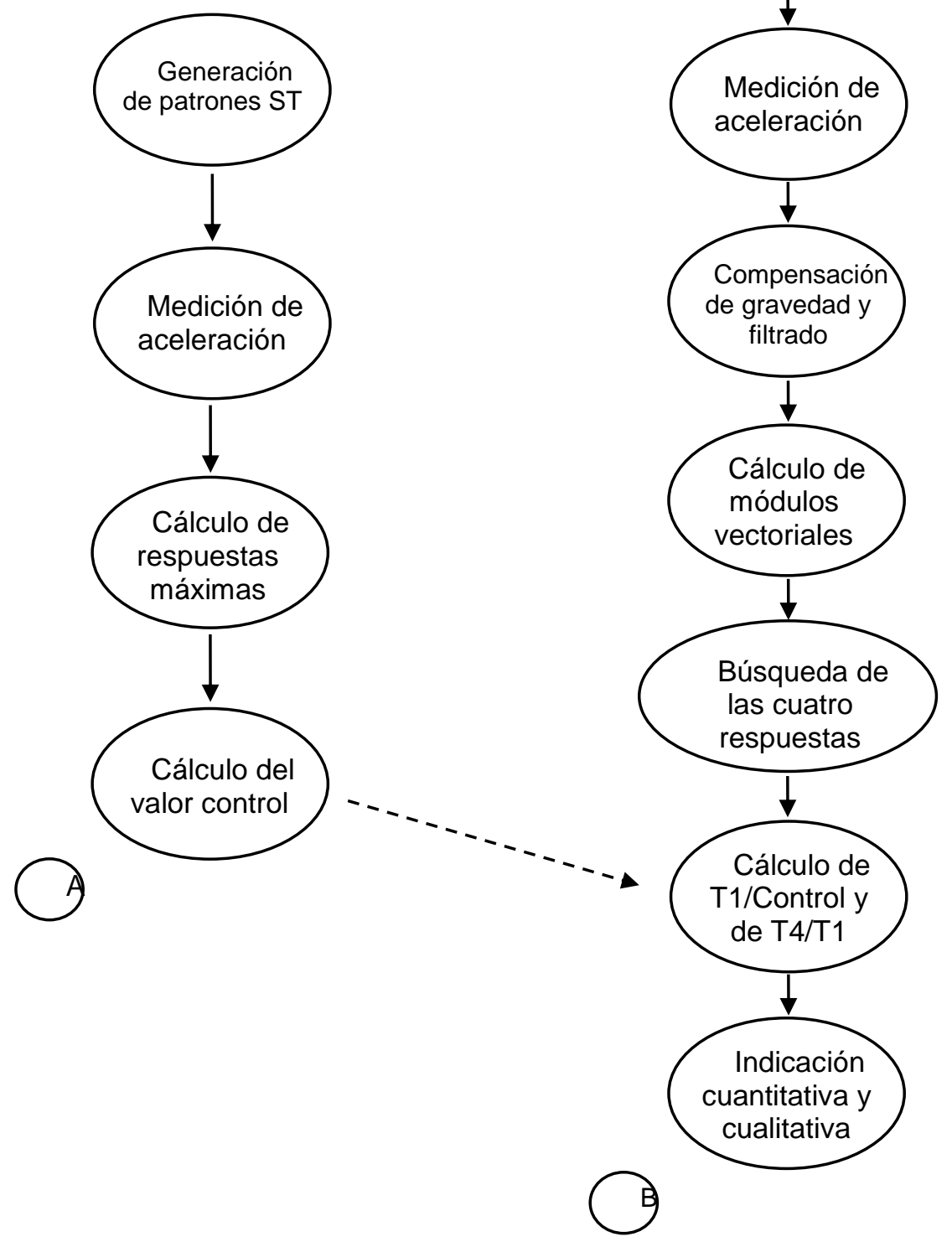

Figura 3. Representación en bloques del algoritmopara la estimación del bloqueo neuromuscular. En ella, se denota por "A" el proceso de calibración y por "B" un evento de monitorización.

Durante el proceso de calibración, que únicamente se realiza antes de iniciarse el acto quirúrgico, el paciente es estimulado con una secuencia de estímulos ST de intensidad supramáxima. Este proceso permite ajustar el sistema de medición a cada paciente particular y obtener una referencia (control) de su conducción neuromuscular en términos de aceleración, antes de que le sea administrado el BNM. Posteriormente, esta referencia se emplea durante el cálculo de los indicadores de relajación muscular. 
Cada evento de monitorización se inicia con la aplicación del estímulo TOF. En cada pulso de estimulación, se realizan mediciones de aceleración en tres ejes ortogonales, se contrarresta la acción de la fuerza de la gravedad, se calculan los valores modulares de aceleración instantánea y se obtienen los cuatro máximos de aceleración correspondientes al patrón TOF, para, finalmente, calcular los siguientes indicadores de relajación muscular:

- T1/Control: Es el porcentaje del cociente entre el valor máximo de la respuesta al primer estímulo del patrón TOF y el valor de referencia obtenido del proceso de calibración. Este indicador toma singular importancia cuando se emplean BNMdespolarizantes, cuya acción provoca la atenuación pareja de las cuatro respuestas al TOF (11).

- T4/T1: Es el porcentaje del cociente entre el valor máximo de la respuesta al cuarto estímulo del patrón TOF y el valor máximo de la respuesta al primer estímulo. Cuando se emplean BNM no despolarizantes, este indicador es suficiente para caracterizar la función neuromuscular (11).

- Conteo de respuestas: En casos de relajación muscular profunda, los valores máximos de las respuestas aparecen atenuados por debajo de cierto umbral de anulación establecido en el algoritmo. En estos casos, se muestra el conteo de la cantidad de respuestas obtenidas para un patrón TOF, ya que no es posible calcular el indicador T4/T1.

\section{Procedimiento experimental}

Para los ensayos experimentales, se escogieron cirugías de corta duración, que incluyeron procedimientos de colecistectomía y plastia de trompas de Falopio con anestesia general y por vía laparoscópica. El estudio tuvo lugar en el Hospital Clínico Quirúrgico "Hermanos Ameijeiras" de La Habana, Cuba. En todos los casos, se explicó el protocolo experimental al paciente para la obtención de su consentimiento informado. También, se pidió a los anestesiólogos que tomaran las decisiones basándose en las herramientas y en los criterios que ordinariamente emplean, pero evaluando críticamente las indicaciones proporcionadas por el monitor, que tendrían únicamente carácter informativo.

Con el objetivo de someter a los pacientes a iguales condiciones de medición, se tomaron medidas como: posición y forma de sujeción de la mano, colocación del sensor de aceleración y posicionamiento de electrodos. La preparación del paciente se ilustra en la Figura 4.

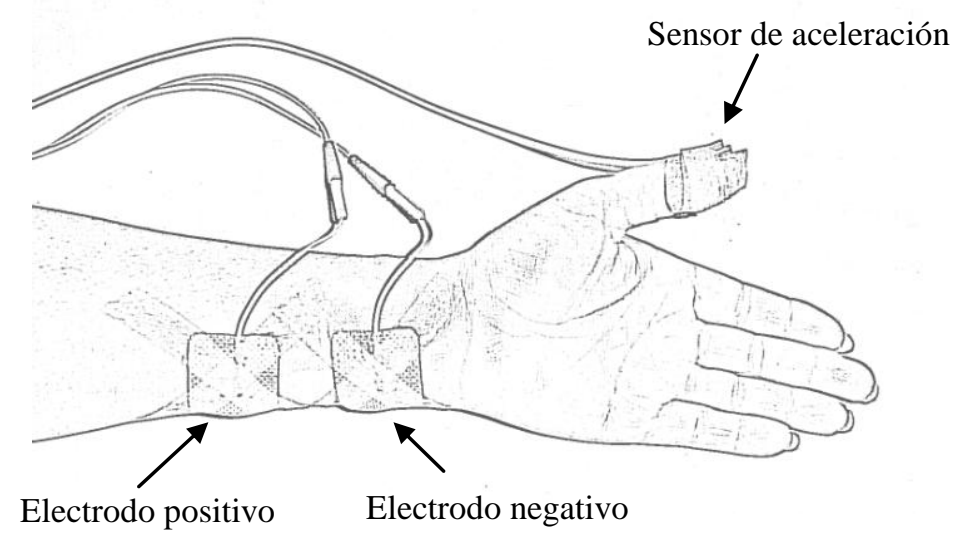

Figura 4: Preparación del paciente durante los ensayos. Se emplearon electrodos autoadhesivos, colocándose el negativo en posición distal y el positivo en posición proximal. El sensor de aceleración se fijó al dedo pulgar con cinta adhesiva hipoalergénica.

Los ensayos se realizaron mediante la estimulación de los nervios mediano y cubital (12), y se registró la respuesta cinética del dedo pulgar. El empleo de electrodos con dimensiones reducidas y su colocación

Cienc. innov. salud. Junio2014; 2 (2):54 - 62. Universidad Simón Bolívar (Col).ISSN: 2344-8636 
sobre el trayecto aproximado de los nervios, tiene la finalidad de aplicar selectivamente el estímulo eléctrico. Se emplearon electrodos autoadhesivos de 9 $\mathrm{cm} 2$, que son apropiados para la estimulación eléctrica transcutánea. Estos electrodos se separaron a una distancia de $3 \mathrm{~cm}$ de borde a borde, colocando el positivo en posición proximal, y el negativo, en posición distal, próximos a la palma de la mano.

En el proceso de calibración, se empleó una intensidad de corriente eléctrica de $50 \mathrm{~mA}$ como mínimo $(6,7,12)$. Durante las etapas de inducción y recuperación (o reversión) de la relajación muscular, el monitor se configuró para monitorización automática cada 15 segundos; y durante el acto quirúrgico, cada 2 minutos. La recogida de los datos se realizó de forma manual, pero en su posterior representación gráfica y análisis, se empleó un software de cálculo.

En todos los pacientes, se realizó la inducción de la anestesia con bolos de propofol a razón de 2 a 2,5 $\mathrm{mg} / \mathrm{kg}$; fentanilo, 2,5 $\mu \mathrm{g} / \mathrm{kg}$; lidocaína, $2 \mathrm{mg} / \mathrm{kg}$; y bromuro de vecuronio, $0,1 \mathrm{mg} / \mathrm{kg}$. Pero, en algunos casos, se empleo una dosis de succinilcolina de 1 $\mathrm{mg} / \mathrm{kg}$ para la intubación. Las dosis de mantenimiento se utilizaron según las necesidades del caso; no obstante, se planificó revertir el bloqueo si no se presentaba recuperación espontánea una vez concluido el acto quirúrgico y cuando el indicador T4/T1 alcanzara valores superiores a $25 \%$.

Vale aclarar que el bromuro de vecuronio es un BNM no despolarizante esteroideo, con un radical similar a la acetilcolina incorporado en el anillo D de su molécula, y sólo uno de los amonios en forma cuaternaria $(3,13-15)$.

Con relación a la cinética y dinámica del fármaco, se señala que su mecanismo de acción compite con la acetilcolina, y se une preferentemente a los receptores nicotínicos postsinápticos de la placa motora, aunque también a los receptores colinérgicos presinápticos. Por otra parte, el bromuro de vecuronio no produce bloqueo del canal iónico, su potencia es de 1 a 1,74 con relación al pancuronio, y es similar al pipecuronio. Se elimina generalmente por el hígado y el riñón, su metabolismo y excreción del compartimento sanguíneo central son indispensables para su recuperación. Su vida de distribución media varía entre 3 y 5 minutos, en tanto que la vida media de eliminación varía entre 60 y 90 minutos. Además, en su presencia, el aclaramiento plasmático demora entre
3 y $5 \mathrm{ml} / \mathrm{min}$, siendo más rápido que el de otros relajantes. Por último, su volumen de distribución varía entre 250 y $350 \mathrm{ml} / \mathrm{kg}$, y la unión a las proteínas plasmáticas es del orden de $25 \%(3,14)$.

Entre las situaciones que modifican la farmacocinética del bromuro de vecuronio, se encuentran los diferentes agentes anestésicos, la edad, el uso concomitante de otros relajantes y el estado ácido básico (3, 14-16).

\section{Resultados}

Se monitorizó la función neuromuscular intraoperatoria en diez pacientes, durante actos quirúrgicos con las características referidas anteriormente. A continuación, se presenta el comportamiento de los cinco casos con resultados más significativos.

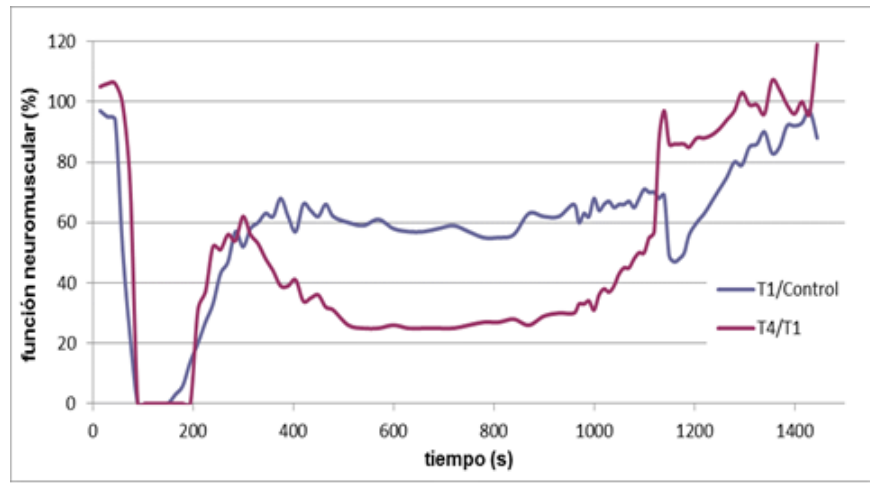

Figura 5. Comportamiento en el tiempo de los indicadores de relajación muscular para el caso ANN

La Figura 5 ilustra el caso ANN con un tiempo anestésico-quirúrgico de 24 minutos. En ella se evidencia el inicio del proceso quirúrgico con indicadores T1/Control y T4/T1 cercanos a 100\%. En ese momento, la inducción de la relajación muscular abrupta mediante succinilcolina para la intubación se reflejó adecuadamente. Ya durante el acto quirúrgico, el indicador T4/T1 se mantuvo por debajo de $40 \%$. La extubación del paciente se efectuó cuando la conducción neuromuscular fue similar a la indicada inmediatamente después del proceso de calibración. 


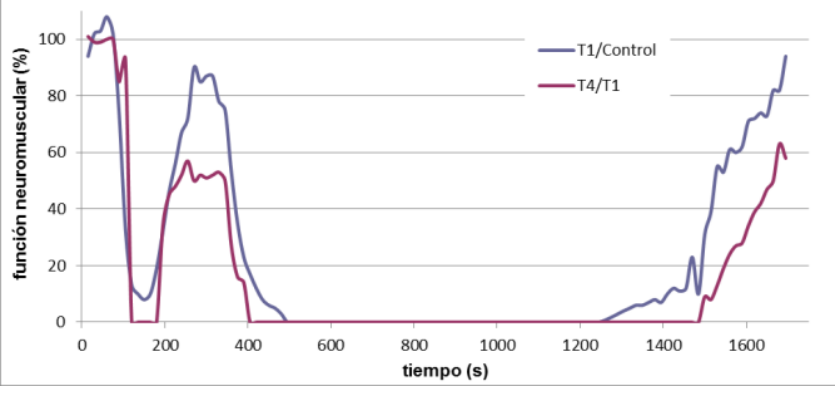

Figura 6. Comportamiento en el tiempo de los indicadores de relajación muscular para el caso YAI

En la Figura 6, se presenta el caso YAI con un tiempo anestésico-quirúrgico de 28 minutos. Antes de inducir la relajación muscular, se aprecian valores próximos a $100 \%$ en los indicadores. Al inicio del acto quirúrgico, se reflejó adecuadamente una recuperación del paciente, así como el efecto de la dosis de mantenimiento administrada. En este caso, se logró una relajación muscular profunda, observándose, en el caso del indicador T4/T1, el cambio de porcentaje a conteo de respuestas, no representado en la Figura. Este paciente mostró dificultad para respirar al ser extubado, lo que concuerda con los indicadores de relajación muscular en ese momento.

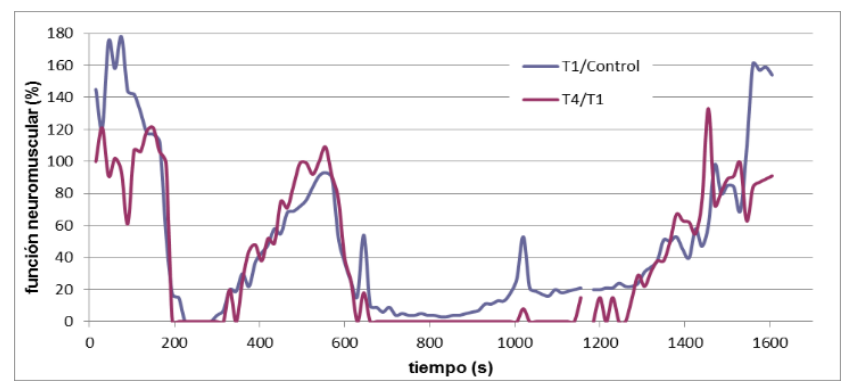

Figura 7. Comportamiento en el tiempo de los indicadores de relajación muscular para el caso MAR

En la Figura 7 se registra el caso MAR con un tiempo anestésico-quirúrgico de 27 minutos. En ella, se observan valores de los indicadores superiores a $100 \%$ antes de la inducción de la relajación, que son comparables con los que se obtuvieron después de la recuperación final del paciente. En este caso, fue necesaria la administración de dosis de mantenimiento tras la inducción inicial. La extubación del paciente se realizó cuando existía criterio para ello, según las indicaciones, justamente con un T4/T1 en el entorno de $90 \%$.

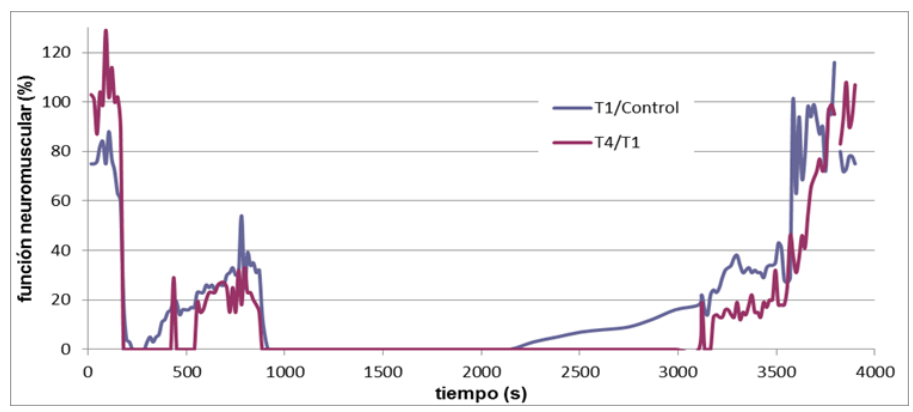

Figura 8. Comportamiento en el tiempo de los indicadores de relajación muscular para el caso LUZ

En la Figura 8, se ilustra el caso LUZ, con un tiempo anestésico-quirúrgico de 65 minutos. El paciente se mantuvo con una relajación muscular profunda durante el procedimiento quirúrgico y se observó el cambio de porcentaje a conteo de respuestas en el indicador T4/T1, lo que no se representa en la Figura. La intubación y la extubación del paciente se realizaron cuando existía criterio para ello, según las indicaciones del monitor. Al comparar los valores finales de los indicadores con los obtenidos inmediatamente después de la calibración inicial, se observa que el paciente se recuperó adecuadamente.

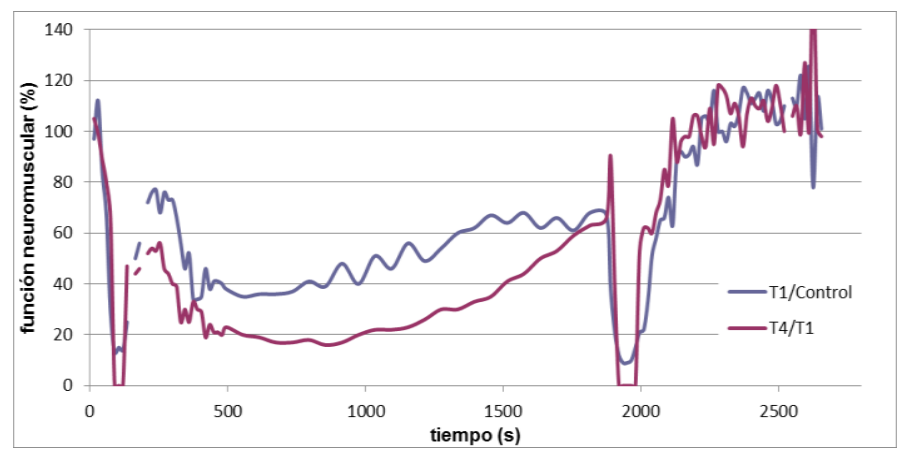

Figura 9. Comportamiento en el tiempo de los indicadores de relajación muscular para el caso ARE

La Figura 9 registra el caso ARE, con un tiempo anestésico-quirúrgico de 44 minutos. 
En este caso, el anestesiólogo mantuvo al paciente en un estado de relajación no profunda durante el transcurso del acto quirúrgico. Y, a solicitud del cirujano, fue necesaria la administración de una dosis adicional de BNM para concluir el acto quirúrgico, eventos que fueron indicados adecuadamente por el monitor. Como en los casos anteriores, la intubación y la extubación del paciente se realizaron cuando existía criterio para ello, de acuerdo con las indicaciones del monitor.

\section{Discusión}

El prototipo del monitor de bloqueo neuromuscular funcionó de forma estable durante diez actos quirúrgicos realizados con instrumental de cirugía video-laparoscópica, lográndose inmunidad ante las interferencias causadas por la electrocirugía.

Se emplearon las dos fuentes de alimentación previstas en el diseño del monitor y se obtuvieron resultados prácticos similares. El empleo de la fuente de alimentación externa de grado médico generó requisitos adicionales de diseño en lo referente a la longitud de su cable y a la solución constructiva del conector para la red de energía. En cambio, la utilización de la batería interna permitió una autonomía del monitor de más de seis horas, pero también generó requisitos adicionales de diseño en lo referente a la implementación de un mecanismo de apagado automático para casos de inactividad del monitor.

A criterio de los especialistas involucrados, en la mayoría de los actos quirúrgicos monitorizados, los indicadores de relajación muscular registrados mostraron significativa correspondencia con la clínica de los pacientes.

Las curvas correspondientes a la variación en el tiempo de los indicadores de relajación muscular exhibieron un comportamiento esperado: valores elevados antes de la inducción de la relajación muscular y al final de los procesos de recuperación o reversión, así como disminución del porcentaje hasta valores cercanos o iguales a cero durante los períodos de relajación muscular del paciente. Para el caso del indicador T4/T1, se observó el cambio de porcentaje a conteo de respuestas en presencia de una relajación muscular profunda. En los casos en que se administró el BNMdespolarizante, el monitor indicó adecuadamente la atenuación pareja a las cuatro respuestas al TOF.

Las ventajas de este tipo de monitorización se evidenciaron en la casuística presentada porque apoyaron la actuación oportuna del anestesiólogo durante las decisiones críticas: intubar, administrar dosis de mantenimiento y extubar. No fue necesario revertir el bloqueo en ningún paciente.

Se ha planteado la necesidad de incorporar nuevas guías o estrategias de buenas prácticas de uso de los BNM (17), pero en la actualidad no es un hecho de obligatorio cumplimiento en anestesiología. Sólo existen recomendaciones de actuación con el propósito de promover la monitorización neuromuscular como una herramienta muy útil para el uso racional de los BNM.

Las recomendaciones en cuanto a monitorización de paciente de la American Society of Anesthesiologists incluyen la monitorización del bloqueo neuromuscular, pero sólo de forma ocasional, o sea, en pacientes cuyo estado clínico lo exija (18).

En síntesis, los resultados son satisfactorios, pero aún preliminares, dado que, a criterio de los autores, debe aumentarse el número de casos y extenderse la monitorización a otros procedimientos quirúrgicos más complejos y de mayor duración. Por ejemplo, a procedimientos en que se emplee otro emplazamiento para las partes aplicables al paciente y otro tipo de equipamiento de electrocirugía. Así se realizará un estudio más completo de correspondencia entre los indicadores que son producto de la monitorización y la clínica real del paciente.

\section{Agradecimientos}

Los autores expresan su reconocimiento a los especialistas del Hospital Clínico Quirúrgico "Hermanos Ameijeiras", que de una forma u otra apoyaron y colaboraron durante la realización de estos ensayos en sus instalaciones. También a los pacientes que, desde esa posición, confiaron y apoyaron esta etapa del desarrollo de un monitor de bloqueo neuromuscular. 


\section{Referencias}

1. Eikermann M, Groeben H, Hüsing J, Peters J. Accelerometryofadductorpollicismusclepredictsre coveryofrespiratoryfunctionfrom neuromuscular blockade. Anesthesiology. 2003; 98(6):13331337.

2. Claudius $\mathrm{C}, \quad$ Viby-Mogensen J. Acceleromyography for use in scientificandclinicalpractice. Anesthesiology. 2008; 108(6):1117-1140.

3. Cordero EI. Monitorización de lafunción neuromuscular. En: Cordero I. Relajantes musculares enla clínica anestesiológica. Editorial Ciencias Médicas, La Habana, 2010. pp. 83-94.

4. Vega D, Forneiro Y. Evaluación de un sistema para lacaracterización de larespuestadel dedo pulgar ante laaplicación de un estímulo eléctrico. $\mathrm{V}$ Latin American CongressonBiomedicalEngineeringCLAIB 2011; IFMBEProceedings 33:718-721.

5. Rodríguez L, Folgueras J, Cordero I, Cartaya ME, Fernández AR. Relax M: monitor - estimulador de diseño nacional. Estudio preliminar ensujetos no anestesiados. Rev cuba anestesiolreanim. 2003; 2(3):1-4.

6. Nuñez N, Vega D, Forneiro Y. Búsquedadel Estímulo Supramáximo para suEmpleoenlaMonitorización de laFunción Neuromuscular. V Latin American CongressonBiomedicalEngineeringCLAIB 2011; IFMBEProceedings 33:842-845

7. KopmanAF, Lawson MilliamperageRequirements

D. SupramaximalStimulationoftheUlnarNervewithSu rfaceElectrodes. Anesthesiology 1984; 61(1):8385.

8. Núñez N, Vega D. Monitor de relajación neuromuscular: una propuesta de diseño. BioingFisMédCub. 2009; 10(3):14-17.

9. Rodríguez L, Folgueras J, Cartaya ME, Ruiz A, Fernández AR, Díaz $M$ et al. Un monitor para determinar laprofundidad de larelajación muscular enanestesiología. Memorias II CongresoLatinoamericano de Ingeniería Biomédica, Habana 2001, artículo 00142, ISSN 950-7132-57-5.

10. Ghai B, Makkar JK, Wig J. Neuromuscular Monitoring: A Review.
JournalofAnaesthesiologyClinicalPharmacology 2006; 22(4):347-356.

11. Kalli I. Neuromuscular blockmonitoring. In: Kirby R, Gravenstein N, Lobato E, Gravenstein J, Eds. ClinicalAnesthesiaPractice, 2nd Edition, W. B. Saunders, 2002. pp. 442-451.

12. Rosenberg H, Greenhow DE. PeripheralNerveStimulator Performance: The Influenceof Output PolarityandElectrodePlacement. Canad. Anaesth. Soc. J. 1978; 25(5):424-426.

13. SavareseJJ. New, Newer, Newestandimaginarymusclerelaxants. InternationalAnesthesiaResearchSociety. CourseLecturesReview 1992; 62-67.

14. FaheyMR, Morris RB, Miller RD, SohnYJ, Cronnelly R, Gencarelli P. ClinicalpharmacologyofORG NC45 (Norcuron): a new nondepolarizingmusclerelaxant. Anesthesiology. 1981; 55(1):6-11.

15. Saitoh Y. Recovery fromvecuroniumisdelayed in patientswithhypercholesterolemia. Can J Anaesth 2006; 53(6):556-561.

16. Sarrafzadeh-Rezaei F, Eddie Clutton R. The posttetaniccountduringvecuronium-induced

neuromuscular blockade in halothaneanaesthetizeddogs. VetAnaesthAnalg 2009; 36(3):246-254.

17. Fabregat López J, Candia Arana C, CastilloMonzón CG. La monitorización neuromuscular y suimportanciaenel uso de los bloqueantes neuromusculares. Rev. ColombAnestesiol 2012; 40(4): 293-303.

18. A Reportbythe American SocietyofAnesthesiologistsTask Force onPostanestheticCare. PracticeGuidelines for PostanestheticCare. Anesthesiology 2002; 96:742-752. 